\title{
Piercing and surface-crack defects in combination extrusion of fasteners
}

\author{
Heng-Sheng Lin ${ }^{1, *}$, Chien-Yu Lee ${ }^{1}$,Wen-Shun $\mathrm{Li}^{1}$, and Cheng-Tsung $\mathrm{Wu}^{1}$ \\ ${ }^{1}$ Department of Mold and Die Engineering, National Kaohsiung University of Applied Sciences, Kaohsiung 807, Taiwan
}

\begin{abstract}
Piercing or surface-crack defect occurs in combination-extruding the support pin. There has been so far insufficient knowledge in avoiding such forming defects in forming the similar products. In this paper, a combination-extrusion experiment, with various combinations of forward and backward extrusion ratios, was conducted to observe the occurrence of the defects. The result shows that the defects occur at low level of both forward extrusion ratio and backward extrusion ratio used in the combination-extrusion process. The low forming level causes the onset of insufficient plastic metal-flow, which is similar to that of inhomogeneous deformation in the unidirectional extrusion or drawing process. A forming limit diagram was constructed and can be used in the defect prediction for the future development of similar products.
\end{abstract}

\section{Introduction}

The extrusion process has been commonly used in forming fasteners. Such forming operation includes forward, backward, and combined forward-backward extrusion. The forming defects with the forward or backward extrusion processes, such as Chevron cracks, have been investigated extensively [1-6]. However, the investigations on the forming defects with combination extrusion, such as piercing or surface-crack defects, are still scarce. Lee et al. [7] report the occurrence of a lapping defect caused by the dead metal in forming a piston-ring with respective forward and backward extrusion. The lapping defect is avoided by controlling the material flow and eliminating the dead metal zone with a novel die design with combination extrusion.

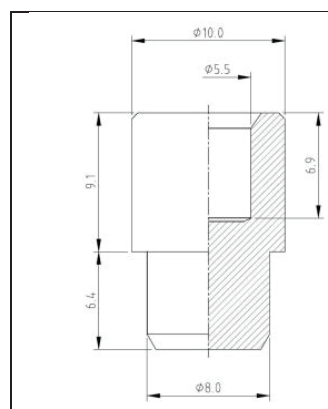

(a)

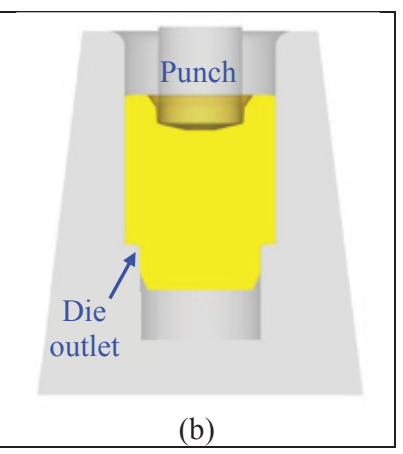

(b)
Fig. 1. (a) Dimensions of support pin; (b) combinationextrusion of support pin.

The demand of the current work arises from the forming design of a support pin, as shown in Fig. 1. The occurrence of the piercing or surface-crack defects, as shown in Fig. 2, renders the combination extrusion as an unreliable operation. The surface cracks may appear sporadically, and be recognized as the forerunner of piercing to happen. Similar approach has been reported by Yoon et al. [8], in their hot bi-directional extrusion with magnesium alloy. There has been so far insufficient knowledge in avoiding such forming defects in forming the similar products.
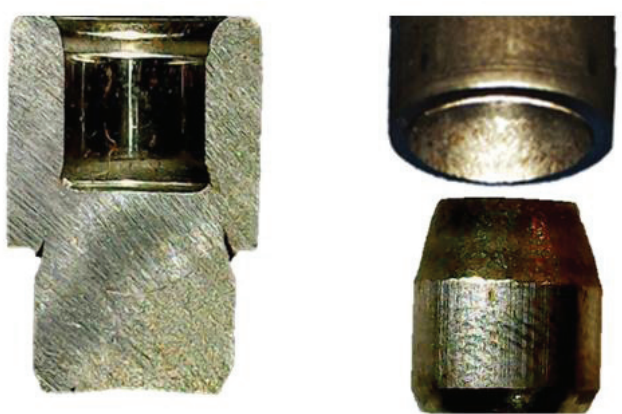

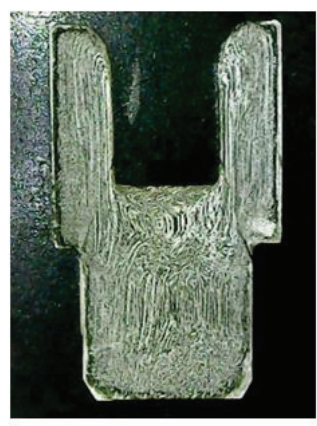

(a)

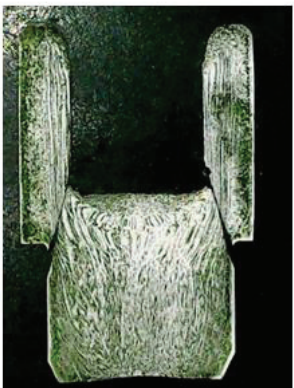

(b)
Fig. 2. Forming defects of combination extrusion: (a) surface crack; (b) piercing.

Corresponding author: hslin@,kuas.edu.tw 
In this paper, a combination-extrusion experiment with various combinations of forward and backward extrusion ratios was conducted to observe the occurrence of the defects. A complete forming limit diagram was also constructed and can be used in the defect prediction for the future development of the similar products. Finite element analysis for effective strain and ductile fracture was performed.

\section{Methods}

\subsection{Experiment}

Annealed ASTM A36 structural steel bar was used as the workpiece material for the combination-extrusion experiment. The Young's modulus was $200 \mathrm{GPa}$, and the Poissson ratio was 0.26 . The workpiece was $25 \mathrm{~mm}$ in diameter and $25 \mathrm{~mm}$ in height, whose size was 2.5 times of the support pin as shown in Fig. 1. The flow-stress of workpiece is shown in Fig. 3. The combination extrusion was conducted on a hydraulic press. The punching speed was $10 \mathrm{~mm} / \mathrm{s}$.

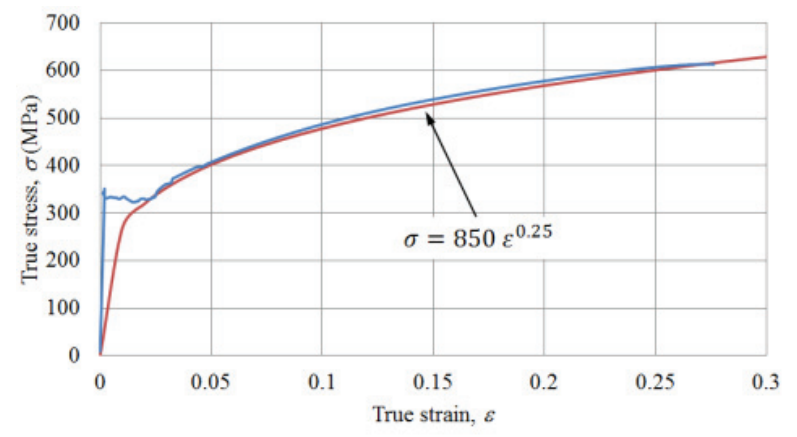

Fig. 3. Flow stress of workpiece.

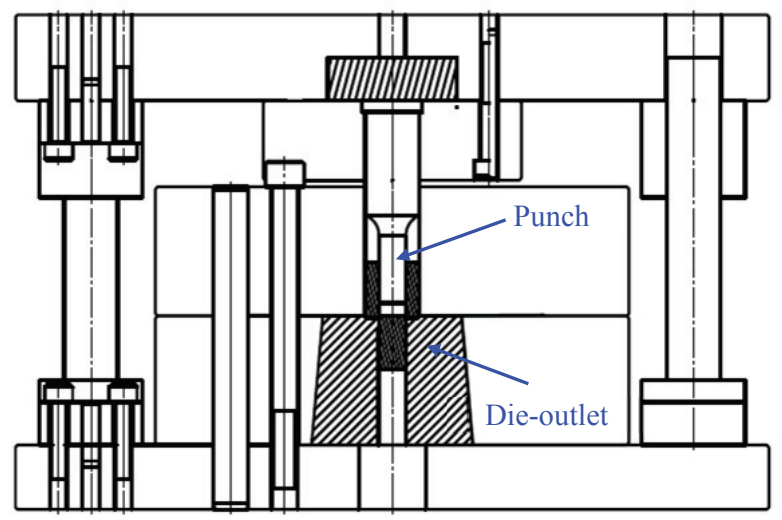

Fig. 4. Die-set configuration of combination extrusion.

Various combinations of forward extrusion ratios and backward extrusion ratios were tested in the experiment. The design of the die set is show in Fig. 4. The forward extrusion ratio is defined as the cross-sectional area of the original workpiece divided by that of the downward extruded part from the die-outlet. The backward extrusion ratio is defined as the cross-sectional area of the original workpiece divided by that of the upward extruded part from the punch. For instance, the forward and backward extrusion ratios in forming the support pin shown in Fig. 1 are

$$
\frac{\frac{\pi}{4} 10^{2}}{\frac{\pi}{4} 8^{2}}=1.56
$$

and

$$
\frac{\frac{\pi}{4} 10^{2}}{\frac{\pi}{4}\left(10^{2}-5.5^{2}\right)}=1.43
$$

, respectively. In the combination-extrusion experiment, three dies of different outlet diameters and three punches of different diameters were used. This will provide various combinations of forward and backward extrusion ratios. The dimensions and design parameters used in the combination-extrusion experiment are shown in Table 1.

Table 1. Dimensions and design parameters used in combination-extrusion experiment.

\begin{tabular}{|l|c|}
\hline Billet height, $\mathrm{mm}$ & 25.0 \\
\hline Billet diameter, $\mathrm{mm}$ & 25.0 \\
\hline Die-outlet diameter, $\mathrm{mm}$ & $12.50(4.00)$ \\
(Forward extrusion ratios are in & $17.68(2.00)$ \\
parentheses) & $21.65(1.33)$ \\
\hline Punch diameter, $\mathrm{mm}$ & $12.50(1.33)$ \\
(Backward extrusion ratios are in & $17.68(2.00)$ \\
parentheses) & $21.65(4.00)$ \\
\hline
\end{tabular}

\subsection{Finite element simulation}

Finite element software DEFORM 2D was used for the analysis. The workpiece was assumed to be elasto-plastic and the punch and die were assumed to be rigid. The flow rule is

$$
\sigma=850 \varepsilon^{0.25}
$$

which was obtained from curve fitting of the tensile test. 15,000 elements were used in the simulation and the friction factor was 0.12 . The effects of strain rate and temperature were neglected. The normalized Cockcroft and Latham damage criterion was used in determining the level of ductile fracture, which is defined as

$$
C=\int_{0}^{\bar{\varepsilon}_{f}} \frac{\sigma^{*}}{\bar{\sigma}} d \bar{\varepsilon}
$$

where $C$ is the critical damage value, $\sigma^{*}$ is the maximum tensile stress, $\bar{\sigma}$ is the effective stress, $\bar{\varepsilon}$ is the effective strain, and $\overline{\varepsilon_{f}}$ is the critical effective strain upon material fracture, respectively. The corresponding damage value upon fracture was 1.3, which was obtained from matching the tensile test for the ASTM A36 structural steel bar of $12.5 \mathrm{~mm}$ in diameter. 


\section{Results and discussion}

\subsection{Experimental result}

Fig. 5 shows the experimental result of the combination extrusion. The corresponding forming limit diagram plotted against the forward and backward extrusion ratios is shown in Fig. 6. In order to explicitly compare the experimental result with the forming limit diagram, the corresponding forward and backward extrusion ratios are reversed on both the horizontal and vertical labels of Fig. 6, respectively. The patterns of flow line formed with the combination-extrusion are similar to those of Fig. 2, in which the piercing cracks are crossing instead of parallel to the metal flow. This indicates that the forming defect was not caused by the discrepancy of metal flow and the dead metal blocked by the die corner.

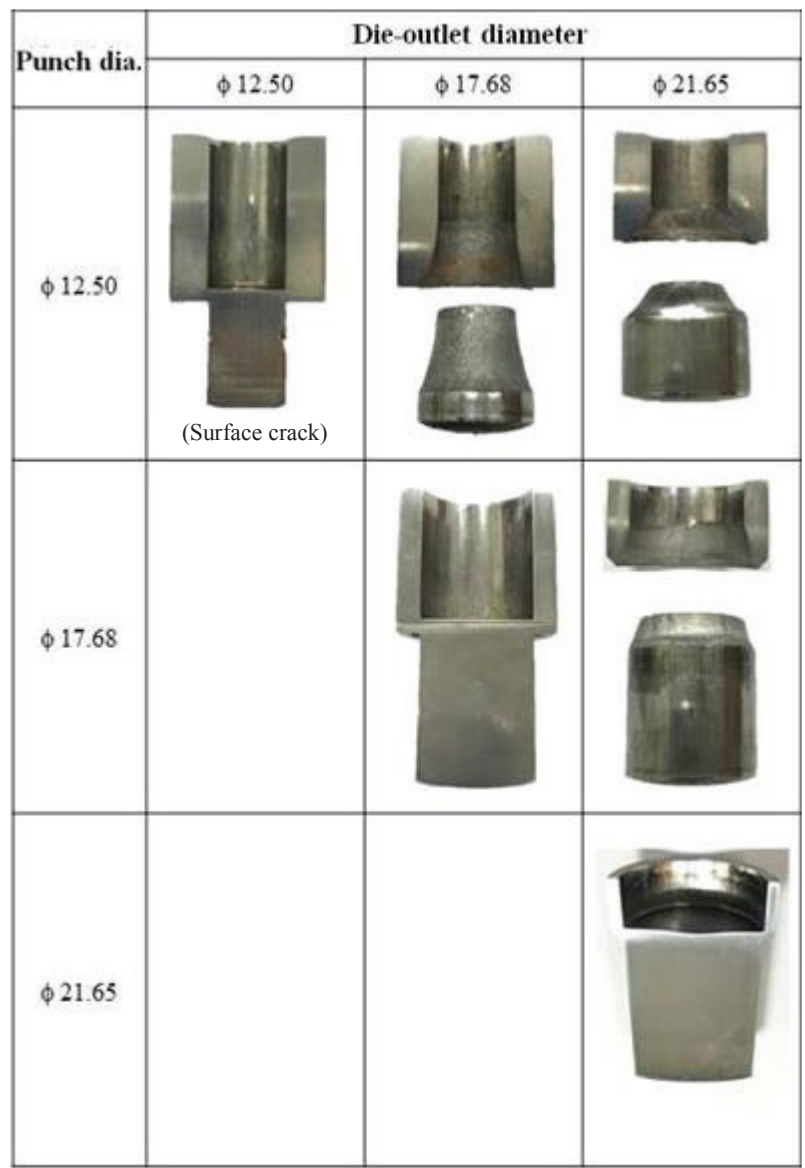

Fig. 5. Cross-section of combination-extrusion experiment.

The experiment shows that satisfactory combination extrusion can be obtained when the die-outlet diameter was small and the punch diameter was large. This translates to a large forward extrusion ratio and a large backward extrusion ratio used during the combination extrusion. Surface-crack defect occurred at a small dieoutlet diameter $(12.5 \mathrm{~mm})$ and a small punch diameter $(12.5 \mathrm{~mm})$, i.e. a large forward extrusion ratio and a small backward extrusion ratio. Piercing defect occurred when the die-outlet diameter was large and the punch diameter was small, i.e. a small forward extrusion ratio and a small backward extrusion ratio, respectively.

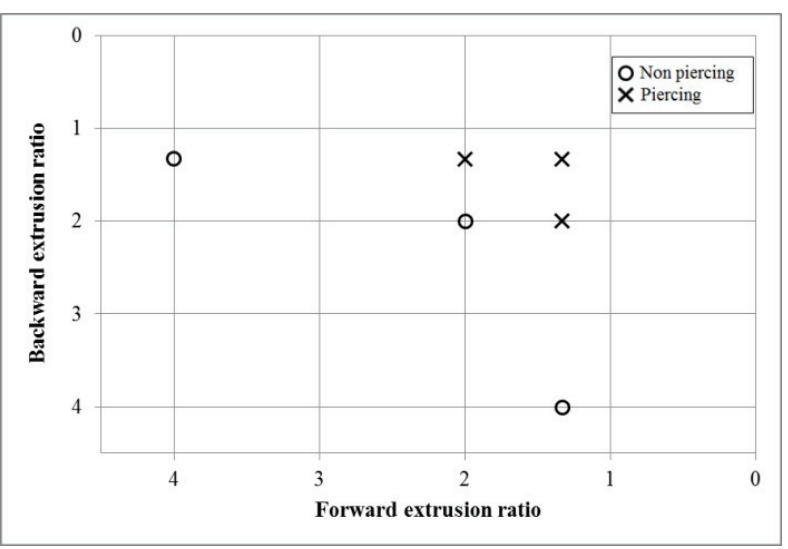

Fig. 6. Forming limit diagram of combination-extrusion experiment.

The result shown in Figs. 5 and 6 is similar to that of Yoon et al. [8]. Contrary to the conventional wisdom, the piercing defect does not necessarily occur when the dieoutlet diameter is close to that of punch. Moreover, the piercing defect does not tend to occur when the consumption of distortion energy is high, i.e. high level of extrusion ratio. Both phenomena indicate that the forming defects occurred in combination extrusion are attributed to insufficient deformation level, which is also a primary factor causing the strain inhomogeneity of workpiece [9] in the forming processes. The low forming level also is an indication that the hydrostatic pressure is low, and the tendency of crack initiation in the workpiece is not suppressed.

\subsection{Simulation result}

Fig. 7 shows the distribution of effective strain obtained from the FEM analysis corresponding to the conditions used in the combination extrusion of Fig. 5. The strain appears concentrated along the workpiece's forwardextruded outer surface and the backward-extruded inner surface when the diameter of the die-outlet is equivalent to that of the punch. This phenomenon might lead to the speculation that piercing or surface defect would occur under such condition attributed to the concentrated deformation. However, the experimental result shown in Fig. 5 reveals that piercing does not occur under such condition. On the contrary, piercing occurs at both low forward and low backward extrusion ratios where the effective strain appears mild and scattered. In this analysis, the distribution of effective strain does not explain well on the forming defects of combination extrusion.

A similar trend is found in the distribution of the normalized Cockcroft and Latham damage values as shown in Fig. 8. The damage values also appear concentrated along the workpiece's forward-extruded outer surface and the backward-extruded inner surface when the diameter of the die-outlet is equivalent to that of the punch. However, for the piercing conditions when both forward and backward extrusion ratios are low, the 


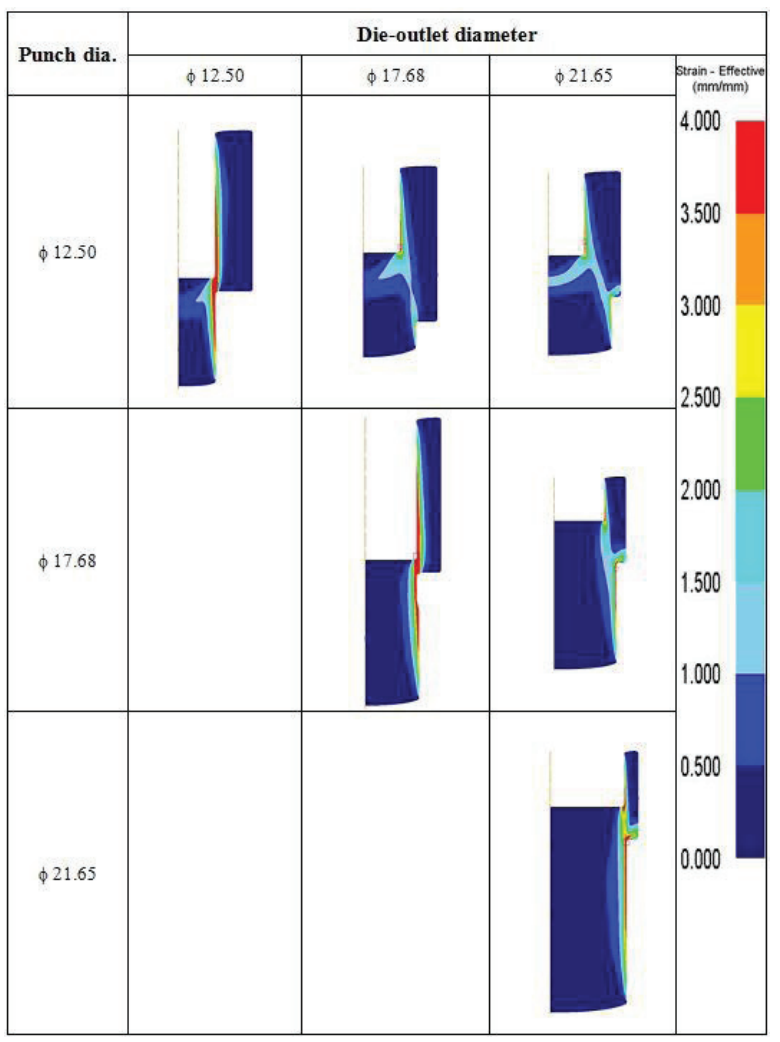

Fig. 7. Distribution of effective strain.

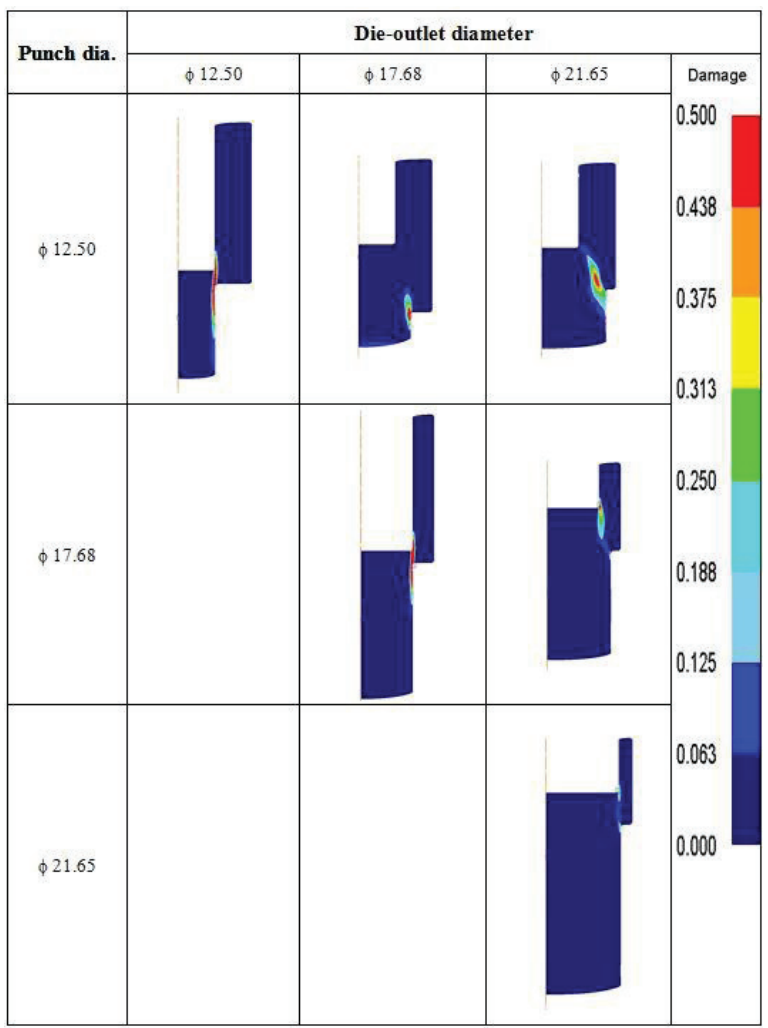

Fig. 8. Distribution of the normalized Cockcroft and Latham damage values. damage values appear smaller and less concentrated. This is no surprise because the damage values are obtained by the integration of the maximum tensile stress and the effective strain. And the effective strain affects the damage values significantly. The damage values were less than 1.3, the critical damage value upon fracture obtained from matching the tensile test. The analysis of damage values does not predict the occurrence of cracks during the combination extrusion. Therefore, the normalized Cockcroft and Latham damage criterion does not apply well in explaining the forming defects of combination extrusion.

\subsection{Improvement of forming defect for support pin}

\subsubsection{Modification of the pre-forming stage}

For the support pin shown in Fig. 1, the forward extrusion ratio and the backward extrusion ratio are 1.56 and 1.43 , respectively. The forming level falls on the border of the piercing and the non-piercing conditions, according to the forming limit diagram of the combination-extrusion experiment shown in Fig. 6. This indicates that possibility of applying the combination extrusion and avoiding the piercing defect, if some modification measures are made.

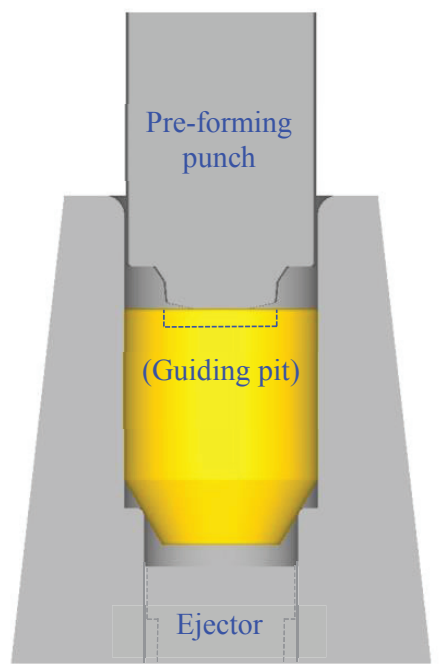

Fig. 9. Die-set configuration of pre-forming stage.

The simple way to avoid the forming defect for the support pin is to increase the corner angles at the die outlet and the punch. But this measure would affect the usability of the product. A quick solution to avoid the forming defect in support pin is to increase the penetration depth of the punch at the pre-forming stage, as shown in Fig. 9. According to the field practice, the purpose of the pre-forming is to provide a shallow circular pit on the back of the workpiece as the guiding pit for the subsequent combination extrusion with the slim punch, as shown in Fig. 1(b). Without the preforming stage, the slim combination-extrusion punch breaks easily because the eccentric buckling might occur 
from the heavy extrusion load. Therefore, by increasing the pre-forming punch stroke slightly further, the bottom side of the workpiece will contact with the ejector and the tendency for backward extrusion will be enhanced. This measure at the same time can significantly distribute the load in the stage of combination extrusion to the pre-forming stage, and the occurrence of piercing defect can be avoided.

\subsubsection{Decomposition of the combination extrusion}

Another robust way to avoid the forming defects in combination extrusion is to decompose the operation into respective forward extrusion and backward extrusion. Although the hydrostatic pressure in the workpiece during the unidirectional extrusion operation is usually very high, the tendency for the crack initiation is suppressed. Punch and die life maybe decreased because of the larger hydrostatic pressure generated in the unidirectional extrusion. The decomposition also requires an extra forming stage and additive tool cost as well as a larger forming press.

Fig. 10 shows the flow lines of the cross-section formed with the unidirectional extrusion. There are neither cracks formed on the corner of the forwardextruded outer surface nor on the corner of the backward-extruded inner surface. Normally the forward extrusion followed by backward extrusion is favored because of the convenience of punch ejection. Otherwise, after the completion of the preliminary backward extrusion, the punch gets wrapped by the workpiece during the following forward extrusion. This causes the risk of breakage on the punch.

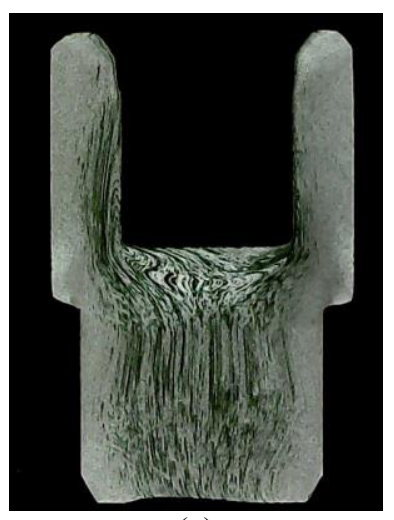

(a)

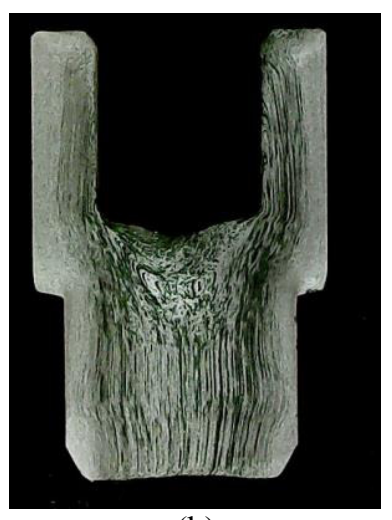

(b)
Fig. 10. Flow line images of the cross-section formed with unidirectional extrusions: (a) forward extrusion followed by backward extrusion; (b) backward extrusion followed by forward extrusion.

\section{Conclusion}

In this paper, a combination-extrusion experiment with various combinations of forward and backward extrusion ratios was conducted to observe the occurrence of forming defects. A forming limit diagram was also constructed and can be used in the defect prediction for the future development of the similar products. Finite element analysis for effective strain and ductile fracture was performed.

(1) The experiment shows that satisfactory combination extrusion can be obtained when the die-outlet diameter was small and the punch diameter was large. This translates to a large forward extrusion ratio and a large backward extrusion ratio used during the combination extrusion. Surface-crack defect occurred at a small die-outlet diameter $(12.5 \mathrm{~mm})$ and a small punch diameter $(12.5 \mathrm{~mm})$, i.e. a large forward extrusion ratio and a small backward extrusion ratio. Piercing defect occurred when the die-outlet diameter was large and the punch diameter was small, i.e. a small forward extrusion ratio and a small backward extrusion ratio, respectively. Contrary to the conventional wisdom, the piercing defect does not necessarily occur when the die-outlet diameter is close to that of punch. Moreover, the piercing defect does not tend to occur when the consumption of distortion energy is high, i.e. high level of extrusion ratio. Both phenomena indicate that the forming defects occurred in combination extrusion are attributed to insufficient deformation level which is also a primary factor causing the strain inhomogeneity of workpiece in the forming processes.

(2) The distribution of effective strain appears concentrated along the workpiece's forward-extruded outer surface and the backward-extruded inner surface when the diameter of the die-outlet is equivalent to that of the punch. This phenomenon might lead to the speculation that piercing or surface defects would occur attributed to the concentrated deformation. However, the experiment reveals that piercing does not occur under such condition. On the contrary, piercing occurs at both low forward and low backward extrusion ratios where the effective strain appears mild and scattered. In this analysis, the distribution of effective strain does not explain well on the forming defects of combination extrusion. A similar trend is found in the distribution of the normalized Cockcroft and Latham damage values. The damage values also appear concentrated along the workpiece's forward-extruded outer surface and the backward-extruded inner surface when the diameter of the die-outlet is equivalent to that of the punch. However, for the piercing condition when both forward and backward extrusion ratios are low, the damage values appear smaller and less concentrated. The damage values were less than 1.3, the critical damage value upon fracture obtained from matching the tensile test. The analysis of damage values does not predict the occurrence of cracks during the combination extrusion.

(3) A quick solution to improve the forming defect in support pin is to increase the penetration depth of the punch at the pre-forming stage. The bottom side of the workpiece will contact with the ejector and the tendency for backward extrusion will be enhanced. This measure at the same time can significantly distribute the load in the stage of combination 
extrusion to the pre-forming stage, and the occurrence of piercing defect can be avoided. Another robust way to avoid the forming defects in combination extrusion is to decompose the operation into separate forward extrusion and backward extrusion. Although the hydrostatic pressure in the workpiece during the unidirectional extrusion operation is usually very high, the tendency for the crack initiation is suppressed.

\section{References}

1. M. Terhorst, A. Feuerhack, D. Trauth, Int J Mater Form, 9(4), 449 (2016)

2. P.O. Bouchard, L. Bourgeon, S. Fayolle, K. Mocellin, Int J Mater Form, 4(3), 299 (2011)

3. K. Komori, Int J Mech Sci, 45,141 (2003)

4. N.V. Reddy, P.M. Dixit, G.K. Lal, Int J Mach Tool Manufact, 40, 95 (2000)

5. P.J. McAllen, P. Phelan, J Mater Process Technol, 183, 210 (2007)

6. K. Saanouni, J.F. Mariage, A. Cherouat, P. Lestriez, Comput Struct, 82, 2309 (2004)

7. D.J. Lee, D.J. Kim, B.M. Kim, J Mater Process Technol, 139, 422 (2003)

8. D.J. Yoon, S.J. Lim, H.G. Jeong, E.Z. Kim, C.D. Cho, J Mater Process Technol, 201, 179 (2008)

9. H.S. Lin, Y.C. Hsu, C.C. Ke, Int J Adv Manuf Technol, 81, 53 (2015) 